\title{
THE IMPOSSIBILITY OF PRODUCING I-131 FROM ITS DAUGHTER Xe-131 BY PHOTONUCLEAR REACTIONS
}

\author{
Tuncay Bayram ${ }^{*}$, Serkan Akkoyun², Necati Çelik³, \\ Emel Haciislamoğlư ${ }^{4}$, Serhat Uruk ${ }^{1}$, Şevki Şentürk ${ }^{1}$
}

\author{
${ }^{1}$ Sinop University, Sinop, Turkey \\ ${ }^{2}$ Cumhuriyet University, Sivas, Turkey \\ 3Gümüşhane University, Gümüșhane, Turkey \\ 4Karadeniz Technical University, Trabzon, Turkey
}

\begin{abstract}
It decays to ${ }^{131} \mathrm{Xe}$ by emitting beta particles. In this study, we have investigated the reproduction possibility of ${ }^{131}$ I from ${ }^{131} \mathrm{Xe}$ by using different reactions, including a photonuclear reaction step. We have used TALYS computer program for calculating cross-sections of the reactions. It was seen that it was not an easy task to reproduce ${ }^{131}$ I from its decay product ${ }^{131}$ Xe because of low reaction cross-section values. According to the results, we have seen that the calculations are in harmony with the TENDL 2014 database.
\end{abstract}

Key words: Photonuclear reaction, iodine, xenon, cross section, TALYS

DOI: $10.21175 /$ RadProc.2016.07

\section{INTRODUCTION}

Photonuclear reactions have been used in nuclear and radiation physics fields [1-4]. A gamma-ray induces a nuclear reaction and the excited target nucleus has an excitation of energy. This reaction is called a photonuclear reaction. In order to get rid of the excess energy, the nucleus emits particles or photons. The most probable way is neutron emission $(\gamma, \mathrm{n})$. This reaction is named a photoneutron reaction. The other possibilities with lower probability are $(\gamma, 2 n),(\gamma, 1 p)$ and $(\gamma, 2 p)$ reactions.

The experimental studies on these reactions have begun in 1934 [5] but there is still a lack of existing data. Therefore, systematic studies of these reactions on different nuclei are needed. There are many examples in using photonuclear reactions, such as nuclear level and half-life identifications, nucleon binding energy determinations, material analysis, radiation protection applications, dosimetry, absorbed dose assessment, activation analysis, radiation transport analyses, physics of fission and fusion reactors, nuclear waste transmutations, and understanding element creations by astrophysical processes can be given as examples to such studies $[2,4]$.

I-131 is a widely used radioisotope in nuclear medicine procedures for diagnostic purpose and treatment of the thyroid gland. It decays to Xe-131 with beta decay. On the other hand, clinical linear electron accelerators can be used for the transformation of the nuclei into other ones. For this reason, a possibility of reproducing $\mathrm{I}-131$ isotope from $\mathrm{Xe}-131$ in nuclear medicine lab is worth to be considered. This may be done by bombardment of Xe-131 with high-energy gamma radiation obtained from clinical electron linear accelerators in radiation oncology department. This subject can be studied in a theoretical framework.

In this work, our main task is studying the possibility for the reproduction of I-131 isotope from its decay product Xe-131. Different paths have been considered for this aim. We have calculated the reaction cross-sections of these paths. According to this, the photo-neutron $(\gamma, n)$, photo-proton $(\gamma, p)$ and $(\gamma, 2 p)$ cross-sections on targets as a function of photon energy have been calculated by using TALYS 1.8 computer program. [6]. Also, in these paths, the neutron- and deuteron-induced reaction steps were necessary in order to reach ${ }^{131}{ }^{1}$ isotope. So, $(n, \gamma)$ and $(d, \gamma)$ reaction cross-sections on related targets as a function of incident particle energy have been calculated. According to the results, it has been seen that the calculations have been consistent with the TENDL 2014 values. [7].

\section{METHOD}

A linux based computer program TALYS has been used for the analysis of the nuclear reactions. The code was written in Fortran computer language. Reactions

t.bayram@ymail.com 
involving neutrons, protons, gamma-rays, deuterons, tritons, helions and alpha particles have been simulated by this program. The incident particle energy range can be used between $1 \mathrm{keV}$ and $200 \mathrm{MeV}$. In the code, the target nuclei mass number can be 5 and heavier. TALYS uses a suitable nuclear reaction model such as optical model, compound nucleus statistical theory, direct reactions and pre-equilibrium processes. For all of the open channels, the reaction cross-section can be calculated in the program. In this study, the default options of the program have been used. TALYS outputs include some information about the nuclear reaction, such as elastic and inelastic scattering cross sections, total cross sections, angular distributions of elastic scattering, angular distributions in discrete levels, cross sections for isomeric and ground states, total particle energy and differential cross sections, emission cross sections, production cross section.

\section{RESULTS AND DISCUSSION}

Three different reaction paths reaching I-131 from Xe-131 are given below in Eqs. (1-3).

$$
\begin{aligned}
& { }^{131} \mathrm{Xe}+\mathrm{\gamma} \longrightarrow{ }^{130} \mathrm{I}+\mathrm{p} \\
& { }^{13} \mathrm{O} \mathrm{I}+\mathrm{n} \longrightarrow{ }^{131} \mathrm{I}+\gamma \\
& { }^{131} \mathrm{Xe}+\mathrm{d} \longrightarrow{ }^{133} \mathrm{Cs}+\gamma \\
& { }^{133} \mathrm{Cs}+\gamma \longrightarrow{ }^{131} \mathrm{I}+2 \mathrm{p} \\
& { }^{131} \mathrm{Xe}+\mathrm{n} \longrightarrow{ }^{132} \mathrm{Xe}+\gamma \\
& { }^{132} \mathrm{Xe}+\mathrm{\gamma} \longrightarrow \quad 131 \mathrm{I}+\mathrm{p}
\end{aligned}
$$

In Eq. (1.a), 131Xe isotope has been induced by photons and emits a proton. 13oI isotope has been produced by this photoproton reaction. Since we are planning to use bremsstrahlung photons with $18 \mathrm{MeV}$ end-point energy from clinical linac accelerators, we have investigated $0-30 \mathrm{MeV}$ energy range before experiments. As can be seen in the Fig.1, the crosssections for this reaction are zero up to $10 \mathrm{MeV}$. Between 10 to $18 \mathrm{MeV}$ energy range, cross-sections increase from $5 \times 10-12$ and $0.09 \mathrm{mb}$. After $18 \mathrm{MeV}$ it is about $0.1 \mathrm{mb}$. The probability of inducement for this reaction is not so high, because $131 \mathrm{Xe}$ isotope is stable and $130 \mathrm{I}$ is radionuclide.

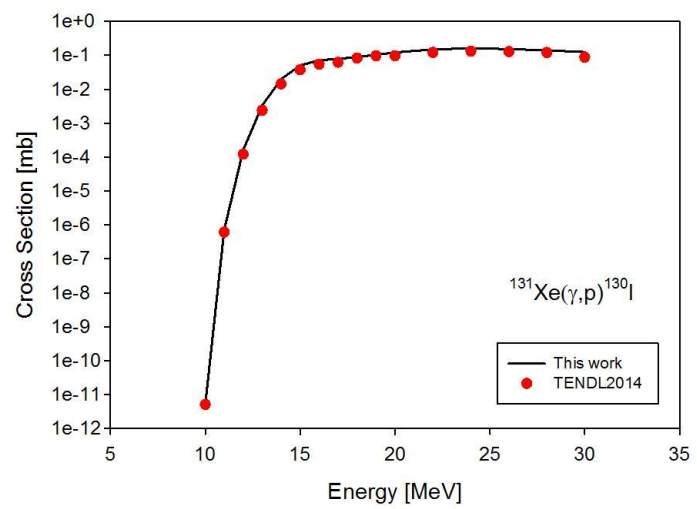

Figure1. Photoproton reaction cross-section on ${ }^{131} \mathrm{Xe}$ target in 10 to $30 \mathrm{MeV}$ energy range
After bombarding ${ }^{130} \mathrm{I}$ by neutrons, ${ }^{131} \mathrm{I}$ can be reproduced by using $(n, \gamma)$ reaction (Eq.1.b). In Fig.2, the reaction cross-sections for this reaction can be seen. It takes maximum value at low energies and starts to decrease with the increase of energy. The reaction cross-section value is high. If one can generate ${ }^{130} \mathrm{I}$ in previous step, it is possible to produce ${ }^{131} \mathrm{I}$.

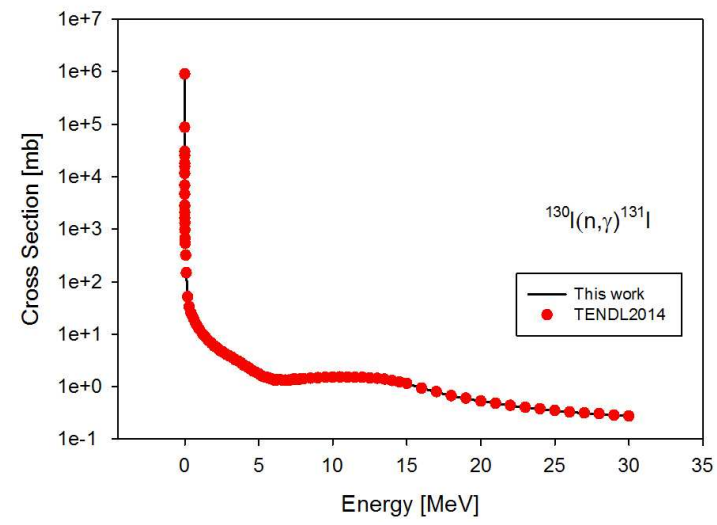

Figure 2. $(n, \gamma)$ reaction cross-section on ${ }^{130} \mathrm{I}$ target in 10 to 30 $\mathrm{MeV}$ energy range

In Eq. (2.a), ${ }^{131} \mathrm{Xe}$ isotope has been induced by deuterons and emits photons. ${ }^{133 C s}$ isotope has been produced by this reaction. As can be seen in the Fig.3, the cross-sections for this reaction are very low with the maximum value of $0.02 \mathrm{mb}$ in $10 \mathrm{MeV}$. Although ${ }^{133} \mathrm{Cs}$ is a stable isotope, it is not so convenient to induce this reaction because ${ }^{131} \mathrm{Xe}$ isotope is also stable and due to Coulomb effect for charged projectiles.

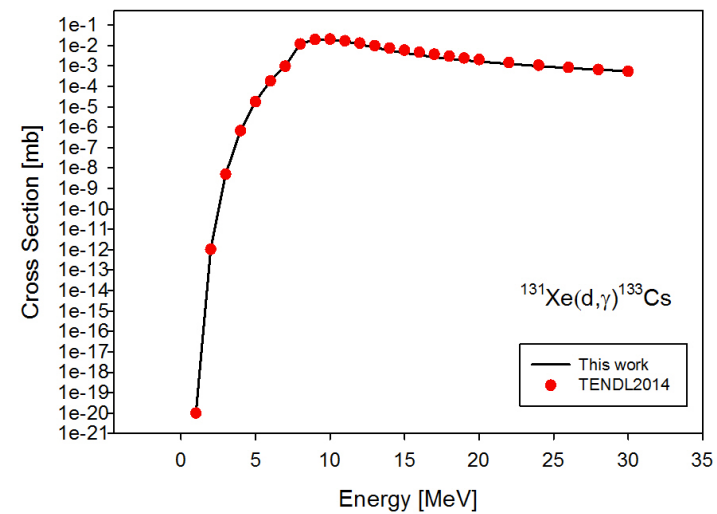

Figure 3. $(\mathrm{d}, \gamma)$ reaction cross-section on ${ }^{131} \mathrm{Xe}$ target in 10 to $30 \mathrm{MeV}$ energy range

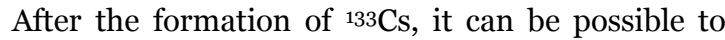
produce ${ }^{131}$ I by $(\gamma, 2 p)$ reaction (Eq.2.b). In Fig.4, the reaction cross-sections for this reaction can be seen. Up to $19 \mathrm{MeV}$, the cross-section is zero. After this value to $30 \mathrm{MeV}$, it is still low about in the order of $10^{-6} \mathrm{MeV}$. Therefore, the production of ${ }^{131} \mathrm{I}$ by this path is not so probable.

The last path for producing ${ }^{131} \mathrm{I}$ is given Eq. (3). According to this, the first step is to generate ${ }^{132} \mathrm{Xe}$ from ${ }^{131}$ Xe by $(n, \gamma)$ reaction (Eq. 3.a). The cross-section 
for this reaction is very high in the low energy region (Fig.5). It takes about constant value of $1 \mathrm{mb}$ after 5 $\mathrm{MeV}$. Due to the fact that both ${ }^{131} \mathrm{Xe}$ and ${ }^{132} \mathrm{Xe}$ isotopes of same element are stable, it is possible to induce this reaction. The other reason for the high probability is the neutral feature of the inducing particle.

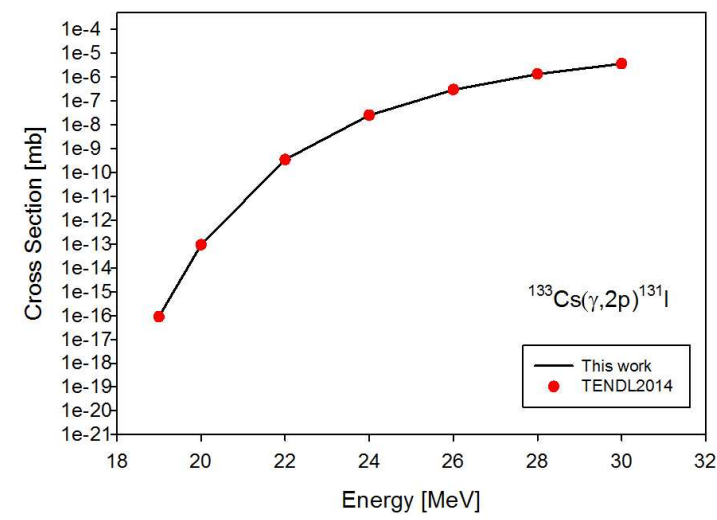

Figure 4. Photoproton reaction cross-section on ${ }^{131} \mathrm{Xe}$ target in 10 to $30 \mathrm{MeV}$ energy range

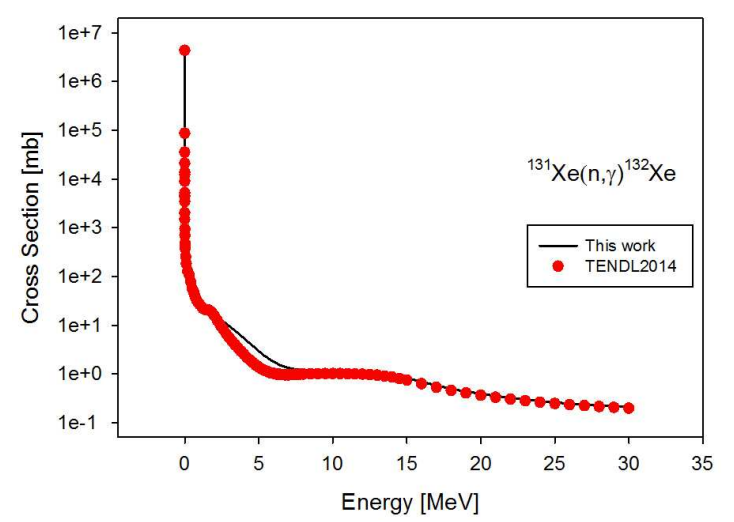

Figure $5 .(n, \gamma)$ reaction cross-section on ${ }^{13}{ }^{1} \mathrm{Xe}$ target in 10 to $30 \mathrm{MeV}$ energy range

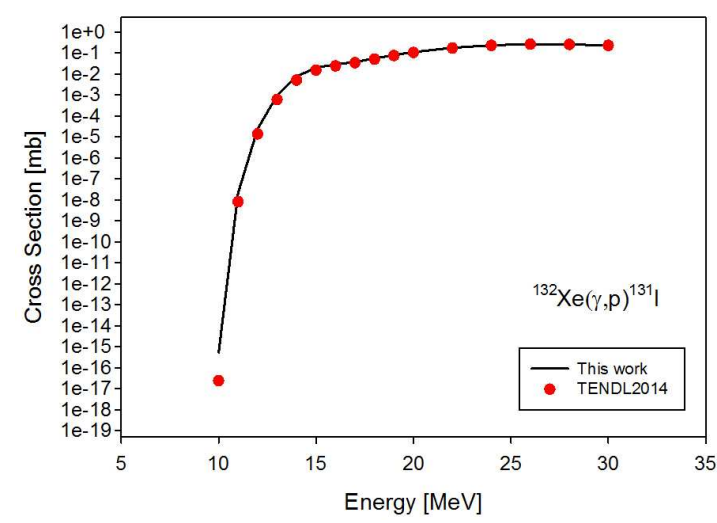

Figure $6 .(\gamma, 2 p)$ reaction cross-section on ${ }^{132} \mathrm{Xe}$ target in 10 to $30 \mathrm{MeV}$ energy range
After bombarding ${ }^{131} \mathrm{Xe}$ by neutrons, ${ }^{131} \mathrm{I}$ can be reproduced by using ( $\gamma, p)$ reaction (Eq.3.b). In Fig.6, the reaction cross-sections for this reaction can be seen. It is zero up to $10 \mathrm{MeV}$ and also very low after this energy value. The probability to produce ${ }^{131} \mathrm{I}$ is low because proton emitting is hindered by Coulomb barrier.

\section{CONCLUSION}

Three different reaction paths for reproducing ${ }^{131} \mathrm{I}$ radioisotope from its decay product ${ }^{131} \mathrm{Xe}$ have been investigated. It was concluded that each of them has a very low probability. In the first path, although the second step is possible, the first step is not possible. In the second path, both steps are impossible. In the last path investigation, the first step is possible but the second is not possible. Therefore, generating ${ }^{131} \mathrm{I}$ from its beta decay daughter is not an easy task.

Acknowledgement: This research has been supported by Sinop University Scientific Research Projects Coordination Unit. Project Number: FEF1901-14-04, 2014.

\section{REFERENCES}

1. M. Schumacher, "Photonuclear reactions," J. Phys. G, vol. 14, suppl., pp. S235-S255, 1988

2. C. Segebade and A. Berger, "Photon Activation Analysis," in Encyclopedia of Analytical Chemistry, R.A. Meyers, Ed., Hoboken (NJ), USA: John Wiley \& Sons, 2008

3. K. Strauch,. "Recent Studies of Photonuclear Reactions," Annu. Rev. Nucl. Sci., vol. 2, pp. 105-128, Dec. 1953

4. IAEA-TECDOS-1178, Handbook on Photonuclear Data for Applications Cross-Sections and Spectra, IAEA, Vienna, Austria, 2000

5. J. Chadwick and M. Goldhaber, "A Nuclear "PhotoEffect': Disintegration of the Diplon by $\gamma$ Rays," Nature, vol. 134, pp. 237-238, Jan. 1934

6. NRG, Petten, the Netherlands and CEA, Bruyères-leChâtel, France, TALYS, software for simulation of nuclear reactions.

Retrieved from: http://www.talys.eu/download-talys/

7. A.J. Koning et al., TENDL-2014: TALYS-Based Evaluated Nuclear Data Library Database, NRG, CCFE, UU, IAEA NDS, Dec. 11, 2014 Retrieved from: ftp://ftp.nrg.eu/pub/www/talys/tendl2014/tendl2014.h $\underline{\mathrm{tml}}$ 